\title{
Localized annular lichen planus on foot mimicking tinea pedis
}

\section{Temiz Selami Aykut', Ozer Ilkay1, Dursun Recep1, Ataseven Arzu1, Fındık Sıddıka², Avunduk Mustafa Cihat $^{2}$}

${ }^{1}$ Department of Dermatology, Necmettin Erbakan Univercity, Meram Faculty of Medicine, Meram/Konya, Turkey, ${ }^{2}$ Department of Pathology, Necmettin Erbakan Univercity, Meram Faculty of Medicine, Meram/Konya, Turkey

Corresponding author: Dr. Temiz Selami Aykut, E-mail: aykutmd42@gmail.com

\begin{abstract}
Lichen Planus (LP) is an inflammatory mucocutaneous disease characterized by typically itchy, pink-purple, polygonal, flat, papules and plaques. The genital region, the oral mucosa, and the flexor surfaces of the extremities are the most common locations of lichen planus. LP usually appears as diffuse lesions. In the literature, single liken planus lesion cases were re ported on the lower lip, esophagus, and breast. Localized foot annular lichen planus is a very rare and extraordinary manifestation of LP. Herein, we present two cases of a single plaque LP on the foot that had been diagnosed as tinea pedis and nummular dermatitis in external family medicine clinics. We want to underline the significance of this uncommon entity in the differential diagnosis of plantar dermatoses in primary care.
\end{abstract}

Key words: Lichen planus; Tinea pedis; Histopathology; Family physician; Primary care

\section{INTRODUCTION}

Lichen Planus (LP) is an inflammatory mucocutaneous disease characterized by typically itchy, pink-purple, polygonal, flat, papules and plaques [1]. The genital region, the oral mucosa, and the flexor surfaces of the extremities are the most common locations of lichen planus [2]. LP usually appears as diffuse lesions [3]. In the literature, single liken planus lesion cases were reported on the lower lip, esophagus, and breast [4-6]. Localized foot annular lichen planus is a very rare and extraordinary manifestation of LP.

Herein, we present two cases of a single plaque LP on the foot that had been diagnosed as tinea pedis and nummular dermatitis in external family medicine clinics. We want to underline the significance of this uncommon entity in the differential diagnosis of plantar dermatoses in primary care.

\section{CASE REPORT}

\section{Case Report One}

A 50-year-old woman presented with erythematous violaceous plaque over the medial side of her left foot (Fig. 1). This lesion had first appeared one year ago. She was diagnosed with tinea pedis and nummular dermatitis in external clinics and was treated with topical antifungal and corticosteroid therapy. The cutaneous examination revealed erythematous violaceous plaques over the medial side of her left foot. There were no additional features in her general physical examination.

In terms of the patient's medical history, she had had type 2 diabetes mellitus for seventeen years and essential hypertension for two years. Laboratory studies were unremarkable.

The histopathological examination revealed focal parakeratosis, an increase in the granular cell layer, and

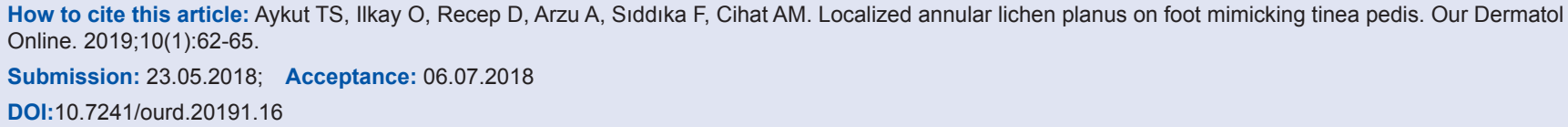


a band-like lymphocytic infiltrate in the dermis (Fig. 2). A periodic acid-Schiff [PAS] stain was negative. The final diagnosis was lichen planus.

$0.05 \%$ clobetasol 17-propionate was given twice daily for ten days, and the patient was advised to take it in the form of occlusion in the evening. After 10 days, tacrolin pomade was recommended to be applied twice a day.

Prior to the study, patient gave written consent to the examination and biopsy after having been informed about the procedure.

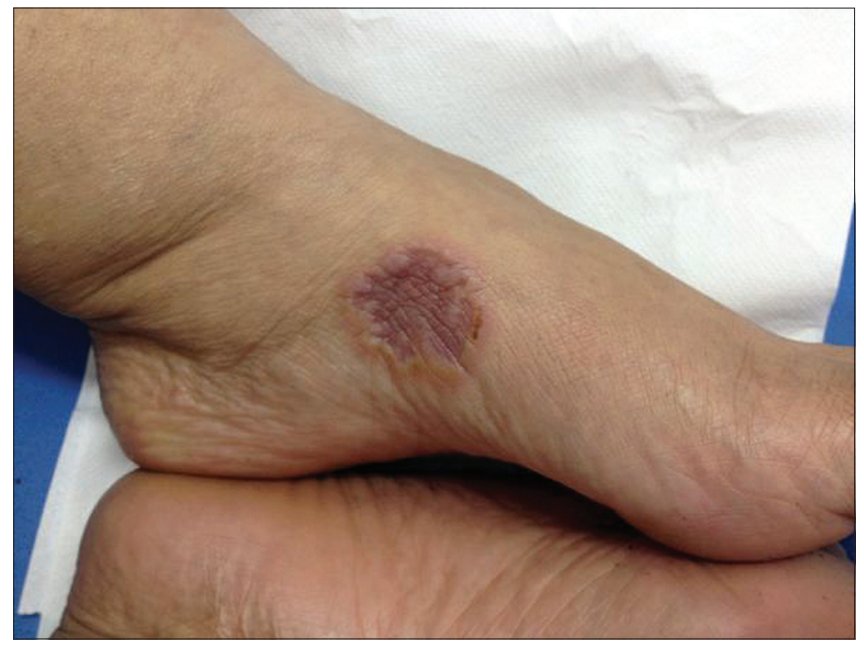

Figure 1: Erythematous violaceous plaque over the medial side of her left foot

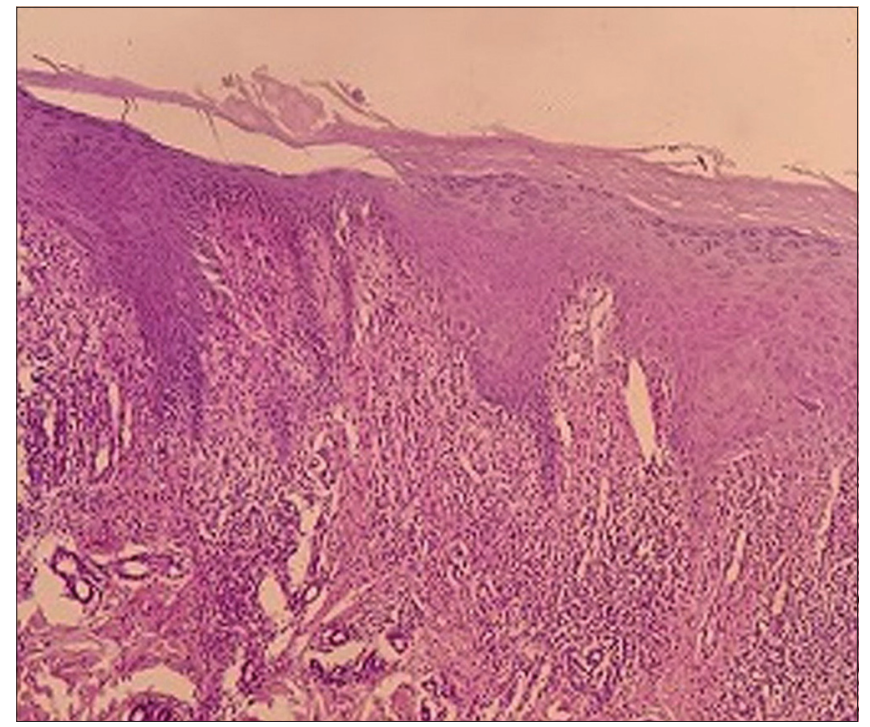

Figure 2: The histopathological examination revealed focal parakeratosis, an increase in the granular cell layer, and a band-like lymphocytic infiltrate in the dermis.

\section{Case Report Two}

A 43-year-old man presented with erythematous violaceous plaque over the medial side of his right foot for six months. He was diagnosed as having tinea pedis in external clinics. The cutaneous examination revealed $3 \times 4 \mathrm{~cm}$ erythematous violaceous plaques over the medial side of his right foot (Fig. 3). His other body areas and mucous membranes were examined and found to be normal. There were no characteristics in the patient's medical history. A native $(\% 10 \mathrm{KOH})$ study was negative for fungal disease.

The histopathological examination revealed focal parakeratosis, an increase in the granular cell layer, and

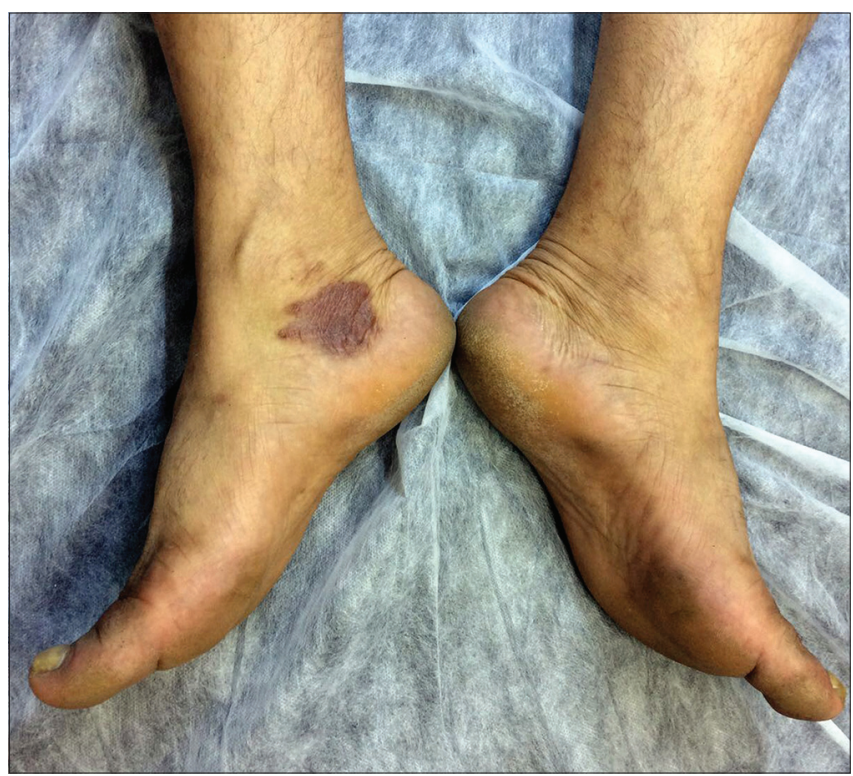

Figure 3: $3 \times 4 \mathrm{~cm}$ erythematous violaceous plaques over the media side of his right foot

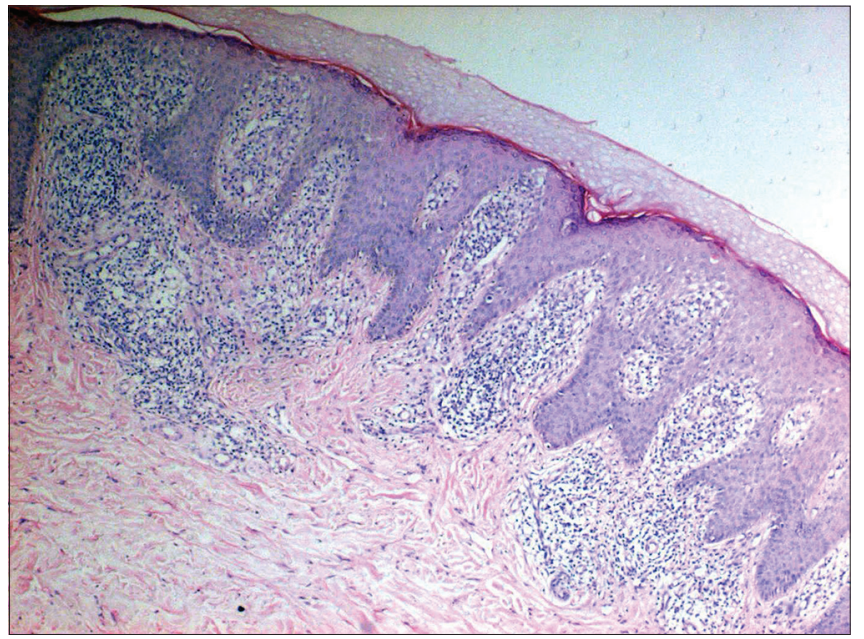

Figure 4: The histopathological examination revealed focal parakeratosis, an increase in the granular cell layer, and a band-like lymphocytic infiltrate in the dermis 
a band-like lymphocytic infiltrate in the dermis (Fig. 4). A periodic acid-Schiff [PAS] stain was negative. The final diagnosis was lichen planus.

$0.05 \%$ clobetasol 17 -propionate was given to the patient twice daily for ten days, and he was advised to take it in the form of occlusion in the evening. After 10 days, tacrolin pomade was recommended to be applied twice a day.

Prior to the study, patient gave written consent to the examination and biopsy after having been informed about the procedure.

\section{DISCUSSION}

Lichen planus lesions can emerge in many different forms: annular, linear, hypertrophic, atrophic, bullous, ulcerative, and pigmented [3]. The lesions in our cases were compatible with annular lichen planus. LP is generally localized to flexures, extremities, genitalia, and oral mucosa [2]. Palmoplantar lichen planus is an uncommon limited variant of lichen planus. In our cases, there was only a single plaque lichen planus on the foot, a rather rare variant of lichen planus [7]. Although the lesion was only on the foot and not on the hands, our cases was thought to be a single plaque lesion as a rare variant of palmoplantar lichen planus.

Lichen planus usually occurs with diffuse lesions anywhere in the body [5]. Lichen planus is mostly acute at its onset [8]. It is quite rare for lichen planus to appear as a single plaque LP. In the literature, single liken planus lesion cases were reported on the lower lip, esophagus, breast, and eyelids [4-6,9]. We did not find a case of a single plaque lichen planus on the foot previously in the literature; thus, our cases were the first reported cases. It was remarkable that the patients were followed for such a long time in primary care with the incorrect diagnosis of tinea pedis. These types of cases can be easily misdiagnosed when they are first referred to the family physician [10].

The differential diagnosis of palmoplantar LP includes psoriasis, callus, tinea, hyperkeratotic eczema, secondary syphilis, verruca, nummular dermatitis, mycosis fungoides, and granuloma annulare [11]. The histopathological examination plays a key role in the differential diagnosis [12]. In our cases, the native examination was negative, and the histopathological examination was compatible with lichen planus.
Acitretin, enoxaparin, cyclosporine, topical cyclosporine, systemic corticosteroid, topical corticosteroid, topical tacrolimus, surgery, UVAl, db-UVB, metronidazole, retinoic acid, sulfasalazine, hydroxychloroquine, mycophenolate mofetil, and thalidomide are the treatment modalities for palmoplantar LP In the literature $[13,14]$. There was also a case of a positive response with topical calcineurin inhibitor therapy reported in the literature $[15,16]$. In our cases, we added tacrolimus cream to a topical steroid therapy, and clinical follow up of our cases continue.

\section{CONCLUSION}

We reported here two cases of a single plaque lichen planus on the foot that had been diagnosed as tinea pedis and nummular dermatitis in external clinics. We present our cases to emphasize the importance of the native examination and histopathology in a differential diagnosis of palmoplantar dermatoses and the rare occurrence of single plaque lichen planus on the foot. Since clinical properties may not be suggestive of LP, a skin biopsy is important for the diagnosis.

\section{CONSENT}

The examination of the patient was conducted according to the Declaration of Helsinki principles.

\section{REFERENCES}

1. Sharma A, Białynicki-Birula R, Schwartz RA, Janniger CK. Lichen planus: an update and review. Cutis. 2012;90:17-23.

2. Schilling L, Vogt T. Lichen ruber planus. Der Hautarzt. 2018;1-7.

3. Gorouhi F, Firooz A, Khatami A, Ladoyanni E, Bouzari N, Kamangar F, et al. Interventions for cutaneous lichen planus (Protocol). Cochrane Database of Systematic Reviews 2009,Issue 4.Art.No: CD008038.

4. Petruzzi M, De Benedittis M, Pastore L, Pannone G, Grassi FR, Serpico R. Isolated lichen planus of the lip. Int J Immunopathol Pharmacol. 2007;20:631-5.

5. Sica M, Zulli C, Manta R, Villanacci V, Conigliaro R, Bassotti G. One-shot balloon dilation of esophageal stricture due to unusual lichen planus localization. J Gastrointestin Liver Dis. 2016;25:427.

6. Palleschi G, Bruscino N, Corradini D, Bassi D, Vega P, Pimpinelli N. Annular lichen planus on the mammary areola: an unusual localization. G Ital Dermatol Venereol. 2016;151:114-5..

7. Ucmak D, Azizoglu R, Harman M. Palmoplantar lichen planus- a report of four cases. J Clin Exp Invest. 2011;2:80-4.

8. Boyd AS, Neldner KH. Lichen planus. J Am Acad Dermatol. 1991;25:593-619.

9. Huang YYM, Wang CM, Potenziani S, Hsu S. Lichen planus of the eyelids: a case report and review of the literature. Dermatol Online J. 2017;23.pii: 13030/qt1c04h08s.

10. Özyurt K, Sucaklı MH, Çölgeçen E, Çelik M. Knowledge of family physicians on common dermatological diseases and their diagnosis 
www.odermatol.com

and management trends. Turkderm Arch Turkish Dermatol Venerol. 2014;48:254-62.

11. Kim MJ, Choi M, Na SY, Lee JH, Cho S. Two cases of palmoplantar lichen planus with various clinical features. J Dermatol. 2010;37:985-9.

12. Landis M, Bohyer C, Bahrami S, Brogan B. Palmoplantar lichen planus: A rare presentation of a common disease. J Dermatol Case Rep. 2008;2:8.

13. Feily A, Yaghoobi R, Nilforoushzadeh MA. Treatment modalities of palmoplantar lichen planus: a brief review. Adv Dermatol Allergol. 2016;33:411

14. Karakatsanis G, Patsatsi A, Kastoridou C, Sotiriadis D. Palmoplantar lichen planus with umbilicated papules: an atypical case with rapid therapeutic response to cyclosporin. J Europ Acad Dermatol
Venereol. 2007;21:1006-7.

15. Ojeda T, Rodríguez-Rey E, Camacho FM. Ulcerative lichen planus of the sole treated with tacrolimus, $0.1 \%$ Actas Dermosifiliogr. 2011;102:383-4.

16. Al-Khenaizan S, Al Mubarak L. Ulcerative lichen planus of the sole: excellent response to topical tacrolimus. Int J Dermatol. 2008;47:626-8.

Copyright by Temiz Selami Aykut, et al. This is an open-access article distributed under the terms of the Creative Commons Attribution License, which permits unrestricted use, distribution, and reproduction in any medium, provided the original author and source are credited.

Source of Support: Nil, Conflict of Interest: None declared. 